(2) Open Access Full Text Article

ORIGINALRESEARCH

\title{
Sarsasapogenin Suppresses RANKL-Induced Osteoclastogenesis in vitro and Prevents Lipopolysaccharide-Induced Bone Loss in vivo
}

This article was published in the following Dove Press journal:

Drug Design, Development and Therapy

\author{
Jiaxuan Peng $\mathbb{D}^{1, *}$ \\ Kangxian Zhao ${ }^{2, *}$ \\ Jiling $\mathrm{Zhu}^{3, *}$ \\ Yanben Wang ${ }^{4,5}$ \\ Peng Sun ${ }^{2}$ \\ Qichang Yang ${ }^{2}$ \\ Tan Zhang ${ }^{4}$ \\ Weiqi $\mathrm{Han}^{4}$ \\ Wenjun $\mathrm{Hu}^{4}$ \\ Wanlei Yang $^{4}$ \\ Jianwei Ruan ${ }^{6}$ \\ Yu Qian ${ }^{1,2,4}$
}

'Guangxi Key Laboratory of Regenerative Medicine, Guangxi Medical University, Guangxi 53002I, People's Republic of China; ${ }^{2}$ The Second Affiliated Hospital and Yuying Children's Hospital of Wenzhou Medical University, Wenzhou, Zhejiang 325000, People's Republic of China;

${ }^{3}$ Department of Clinical Medicine, Medical College of Shaoxing University, Shaoxing,

Zhejiang 3 I2000, People's Republic of

China; ${ }^{4}$ Department of Orthopaedics,

Shaoxing People's Hospital, Zhejiang University School of Medicine, Shaoxing 312000 , Zhejiang, People's Republic of

China; ${ }^{5}$ Department of Orthopaedics, Sir

Run Run Shaw Hospital, School of

Medicine, Zhejiang University, Hangzhou,

Zhejiang, People's Republic of China;

${ }^{6}$ Department of Orthopaedics, Taizhou

Municipal Hospital, Taizhou 318000,

Zhejiang, Republic of China

*These authors contributed equally to this work

Correspondence: Yu Qian

Department of Orthopaedics, Shaoxing

People's Hospital, Zhejiang University

School of Medicine, Shaoxing 312000,

Zhejiang, People's Republic of China

Email doctor 120@hotmail.com
Introduction: Osteoclasts are giant polynuclear cells; their main function is bone resorption. An increased number of osteoclasts and enhanced bone resorption exert significant effects on osteoclast-related bone-lytic diseases, including osteoporosis. Given the limitations of current therapies for osteolytic diseases, it is urgently required to develop safer and more effective alternatives. Sarsasapogenin, a major sapogenin from Anemarrhena asphodeloides Bunge, possesses potent antitumor effects and inhibits NF- $\kappa \mathrm{B}$ and MAPK signaling. However, the manner in which it affects osteoclasts is unclear.

Methods: We investigated the effects of anti-osteoclastogenic and anti-resorptive of sarsasapogenin on bone marrow-derived osteoclasts.

Results: Sarsasapogenin inhibited multiple RANKL-induced signaling cascades, thereby inhibiting the induction of key osteoclast transcription factor NFATc1. The in vivo and in vitro results were consistent: sarsasapogenin treatment protected against bone loss in a mouse osteolysis model induced by lipopolysaccharide.

Conclusion: Our research confirms that sarsasapogenin can be used as a new treatment for osteoclast-related osteolytic diseases.

Keywords: sarsasapogenin, osteoclast, osteoclastogenesis, NF-кB, MAPK, NFATc1, therapeutics

\section{Introduction}

Bone homeostasis is regulated by osteoclasts and osteoblasts. ${ }^{1}$ Enhanced bone resorption and osteoclast numbers in bone remodeling are manifestations of various osteolytic diseases, including osteoporosis-, ${ }^{2}$ cancer- ${ }^{3}$ and osteoarthritis ${ }^{4}$-related bone destruction, which pose a serious health concern. However, currently available anti-osteoporosis drugs often have severe side effects, including musculoskeletal pain, esophageal cancer, ocular inflammation, osteonecrosis of the jaw, and atypical fractures. $^{5-7}$ Therefore, it is required to develop safe alternative drugs that treat osteolytic diseases.

Monocyte precursor cells fuse to form osteoclasts, which possess absorptive capacity. ${ }^{8}$ Osteoclasts express TRAP (tartrate-resistant phosphatase) that attaches to the bone surface through actin-binding sealing zone formation, in which proteases, such as tissue protease $\mathrm{K}$, are released, leading to the degradation of the mineralized bone matrix. The central regulators of osteoclasts differentiation are the two cytokines: macrophage-colony-stimulating factor (M-CSF) and receptor activator of nuclear factor- $\kappa \mathrm{B}$ ligand (RANKL). ${ }^{9,10} \mathrm{M}-\mathrm{CSF}$ is a prerequisite for cell 
proliferation and survival in monocyte cell lines. It also promotes the differentiation of bone marrow precursors and increases the expression of RANKL in bone marrow cells. RANKL is the basic cytokine required to induce monocyte fusion, and monocyte fusion is necessary to form multinucleated osteoclasts. Osteoclastogenesis occurs when the RANK receptor is activated by its ligand RANKL. The combination of RANKL and RANK activates a signal cascade that eventually leads to NFATc1 expression, which is the important factor regulating osteoclastogenesis. $^{11,12}$ The PI3K/Akt, MAPK/AP-1 and NF- $\kappa B$ pathways are activated by RANKL/RANK. These pathways also regulate NFATc $1 .{ }^{13-15}$

LPS (Lipopolysaccharide) is a membrane component of Gram-negative bacteria. It is an effective inducer of inflammatory response, stimulating macrophages, lymphocytes and fibroblasts to produce pro-inflammatory and osteoclast factors like TNF- $\alpha$ and RANKL to promote the osteoclasts recruitment and formation. LPS also has a direct positive effect on osteoclast survival and bone resorption function.

In recent years, many studies have found that natural compounds have potential therapeutic effects on osteoclast mediated bone resorption and osteolytic diseases. Sarsasapogenin (Figure 1A) is a sapogenin present in the Chinese medical herb Anemarrhena asphodeloides Bunge. Sarsasapogenin exerts diverse biological actions, including anti-oxidation, anti-cancer and anti-inflammatory effects; ${ }^{16-19}$ it was also found to suppress the NF-אB pathway in inflammation. ${ }^{17}$ Bao et al have proved the apoptotic effect of sarsasapogenin on HepG2 human hepatoma cells: sarsasapogenin induced the apoptosis of HepG2 cells ${ }^{20}$ Lim et al have investigated the anti-inflammatory effects of sarsasapogenin in vitro and in vivo. Sarsasapogenin potently inhibited NF- $\mathrm{BB}$ and MAPK activation, as well as I $\mathrm{B} \alpha \boldsymbol{\alpha}$ phosphorylation in LPS-stimulated macrophages. ${ }^{17}$ More studies have also shown that its derivatives are effective in anti-inflammatory, neuroprotective, and memory impairment related to aging. ${ }^{21,22}$ However, the role of sarsasapogenin in osteoclast formation and bone resorption has not been reported. Thus, the effects of sarsasapogenin on these areas remain to be studied. We therefore hypothesized whether sarsasapogenin has an inhibitory effect on osteoclastogenesis and prevents bone loss through the NF- $\mathrm{kB}$ and JNK/MAPK signaling pathways. The findings of the present study demonstrated that sarsasapogenin exerted inhibitory effects on osteoclastogenesis, preventing bone loss through the NF- $\mathrm{KB}$ and JNK/MAPK signaling pathways. Our study provides evidence for sarsasapogenin as a potential therapeutic agent in osteoclast-related osteolytic disease.

\section{Materials and Methods Media and Reagents}

Sarsasapogenin was obtained from MCE (New Jersey, USA). It was dissolved in dimethylsulfoxide (DMSO). Alpha-modified minimal essential medium ( $\alpha$ MEM), fetal bovine serum (FBS), and dual antibiotics (penicillin/streptomycin) were obtained from Thermo Fisher (Waltham, MA, USA). RANKL and M-CSF were obtained from R\&D Systems (Minneapolis, MN, USA). Primary antibodies against PI3K (\#4249), Akt (\#9272) and p-Akt (\#4060), ERK (\#4695) and p-ERK (\#4370), p38 (\#8690) and p-p38 (\#4511), p65 (\#8242) and p-p65 (\#3033), JNK (\#9252) and p-JNK (\#9255), IкB $\alpha$ (\#4814), NFATc1 (\#8032), and $\beta$-actin (\#3700) were purchased from Cell Signaling Technology (1:1000; Danvers, MA, USA). A Leukocyte Acid Phosphatase Staining kit (SigmaAldrich, St. Louis, MO, USA) was used to test TRAP enzymatic activity. Remaining supplies were obtained from Sigma-Aldrich unless other explanations.

\section{Cell Culture}

Primary murine-derived bone marrow macrophages (BMM) were collected from the entire bone marrow of 6-week-old male C57BL/6 mice. ${ }^{23}$ Cells were flushed out from femur and tibia bone marrows; then, they were cultured in the complete $\alpha$ MEM medium which contained $10 \%$ FBS, $1 \%$ antibiotic mixture (penicillin and streptomycin) and M-CSF $(25 \mathrm{ng} / \mathrm{mL})$. Then they were placed in a constant temperature incubator at $37{ }^{\circ} \mathrm{C}, 5 \% \mathrm{CO} 2$ until the cell fusion rate reaches more than $90 \%$.

\section{Cytotoxicity Assay}

The cytotoxic effect of sarsasapogenin on BMMs was evaluated by colorimetric 3-(4,5-dimethylthiazol-2-y1)-2,5-diphenyltetrazolium bromide (MTT) assay in accordance. BMMs were inoculated in 96 -well plates in triplicate $\left(8 \times 10^{3}\right.$ cells/well) in complete $\alpha \mathrm{MEM}$ and cultured for $24 \mathrm{~h}$. Following this, they were treated with sarsasapogenin $(1,2,4$, or $8 \mu \mathrm{M})$ for 48 or $96 \mathrm{~h}$. When the above processing was complete, the MTT mixture was added and the plate was further incubated for $4 \mathrm{~h}$. Subsequently, the original medium was replaced with $150 \mu \mathrm{L}$ of DMSO to 
A

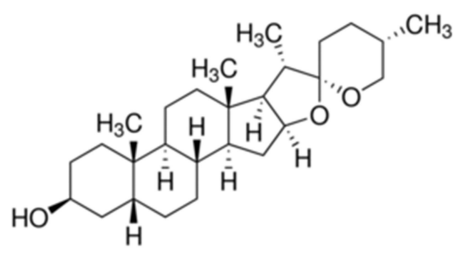

Sarsasapogenin
B

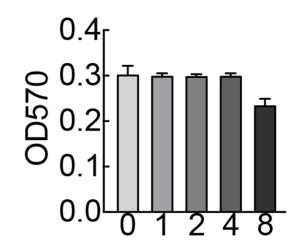

Sarsasapogenin $(\mu \mathrm{M})$ 96h

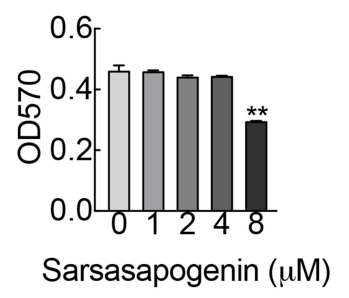

C
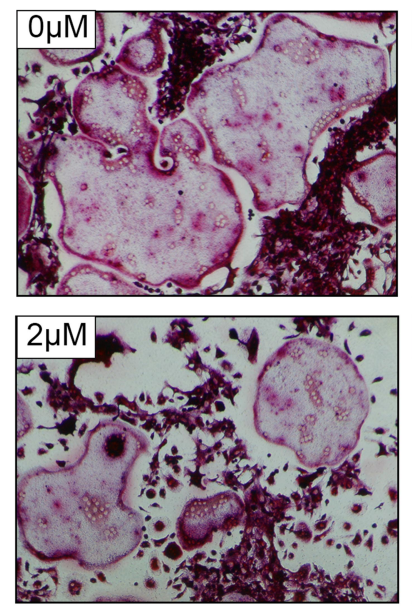

E
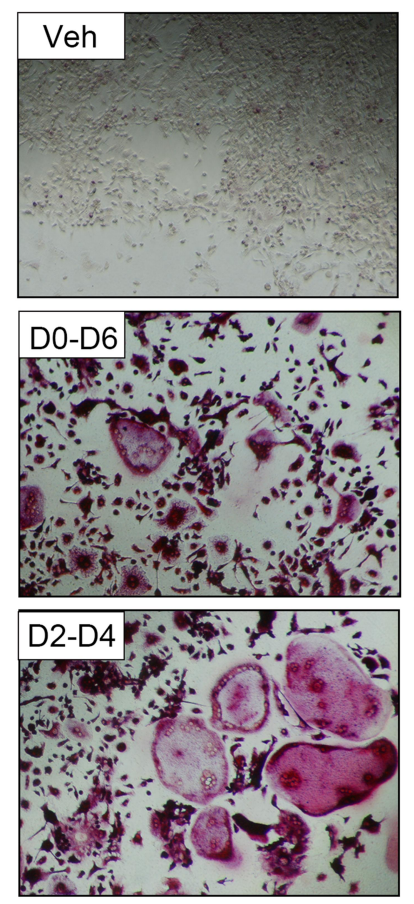
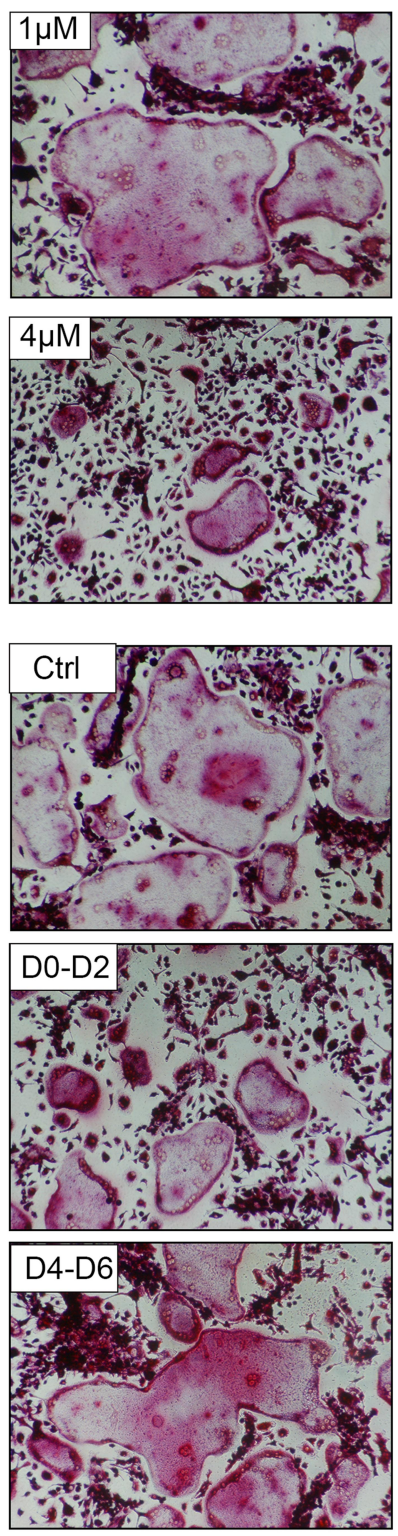

D

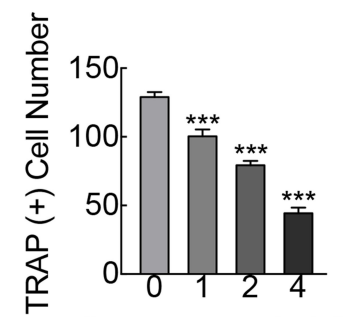

Sarsasapogenin $(\mu \mathrm{M})$

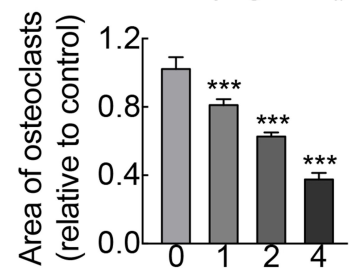

Sarsasapogenin $(\mu \mathrm{M})$

$\mathbf{F}$

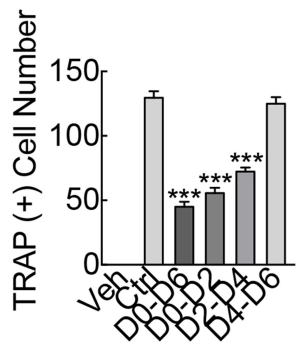

Sarsasapogenin $(4 \mu \mathrm{M})$

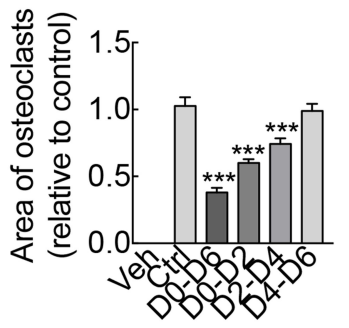

Sarsasapogenin $(4 \mu \mathrm{M})$

Figure I Sarsasapogenin attenuates RANKL-induced osteoclast formation in vitro. (A) Chemical structure of sarsasapogenin. (B) Viability of M-CSF-dependent BMMs following treatment with sarsasapogenin for $48 \mathrm{~h}$ or $96 \mathrm{~h}$ as assessed by MTT assay $(\mathrm{n}=3$ ). (C) Representative images showing the dose-dependent effect of sarsasapogenin on osteoclast formation. BMMs stimulated with $100 \mathrm{ng} / \mathrm{mL}$ RANKL in the absence or presence of indicated concentrations of sarsasapogenin for 7 days were fixed and stained for TRAP activity (magnification $=100 x$; scale bar $=100 \mu \mathrm{m})$. (D) Numbers and area of TRAP-positive multinucleated osteoclasts with more than 3 nuclei were quantified by Imagej $(n=3)$. $(E)$ Representative images showing the time-dependent effect of sarsasapogenin on osteoclast formation. BMMs stimulated with RANKL and treated with $4 \mu M$ sarsasapogenin on the indicated days were fixed and stained for TRAP activity (magnification $=100 x$; scale bar $=100 \mu \mathrm{m})$. $(\mathbf{F})$ Numbers and area of TRAP-positive multinucleated osteoclasts with more than 3 nuclei were quantified by ImageJ $(n=3)$. Bar graphs present the mean $\pm S D ; * * p<0.01$, and $* * * p<0.001$ versus RANKL-only treated control. 
solubilize the formazan crystals. The absorbance was measured at $570 \mathrm{~nm}$ using a microplate reader (BioTek Instruments, Winooski, VT, USA).

\section{Osteoclast Formation and TRAP Staining} After being seeded in triplicate in a 96-well plate, M-CSFdependent BMMs $\left(8 \times 10^{3}\right.$ cells/well $)$ were treated with complete $\alpha$ MEM supplemented with M-CSF $(25 \mathrm{ng} / \mathrm{mL}$ ) and RANKL $(100 \mathrm{ng} / \mathrm{mL})$ with various concentrations of sarsasapogenin $(0,1,2$, or $4 \mu \mathrm{M})$ to concomitantly assay its effects at various concentrations. RANKL-stimulated BMMs were treated with $4 \mu \mathrm{M}$ sarsasapogenin on the stages as follows: early stage, day 0-day 2 (D0-D2); mid stage, day 2-day 4 (D2-D4); late stage, day 4-day 6 (D4D6); whole stage, day 0-day 6 (D0-D6) to measure its effects on different stages of osteoclast formation. Nontreated BMMs and BMMs only treated with RANKL served as negative and positive controls, respectively. Following these treatment procedures, the cells were first fixed with $4 \%$ paraformaldehyde (PFA) and washed with PBS; then, they were stained for TRAP activity. Large multi-nuclear "pancake" shape and obvious TRAP activity were used as the identification basis for mature osteoclasts. The number and area of mature osteoclasts with more than three nuclei were counted.

\section{Bone Resorption}

Equal number of pre-osteoclasts (small osteoclasts that appeared in the early stage of culture) seeded onto discs of bovine bone were stimulated with RANKL (100 ng/mL) under various concentrations of sarsasapogenin $(0,1,2$, or $4 \mu \mathrm{M})$ for 3 days. After incubation period, cells were removed with $6 \%$ sodium hypochlorite. Resorption pits of the bone discs were scanned using scanning electron microscopy (SEM). SEM images were acquired using FEI Quanta 250 Scanning Electron Microscope (Thermo Fisher Scientific, MA, USA). Any pits appearing were counted to the area of bone resorption. ImageJ was used to quantify the resorption area.

\section{F-Actin and DAPI Staining}

BMMs were seeded in a 96 -well plate $\left(8 \times 10^{3}\right.$ cells/well), each drug concentration was repeated for three wells. They were then treated with complete $\alpha$ MEM supplemented with M-CSF $(25 \mathrm{ng} / \mathrm{mL})$ and RANKL $(100 \mathrm{ng} / \mathrm{mL})$ with or without sarsasapogenin as mentioned previously. After the formation period, cells were fixed for 25 minutes using $4 \%$ PFA, and permeated for 5 minutes using $0.1 \%$ Triton
X-100 in PBS. Then they were stained with Anti-stain ${ }^{\mathrm{TM}}$ 488 Fluorescent Phalloidin (Cytoskeleton Inc., Denver, CO, USA) at $20-25^{\circ} \mathrm{C}$ for about $30 \mathrm{~min}$, protected from light. A volume of $1 \mu \mathrm{g} / \mathrm{mL}$ DAPI was used to stain the nuclei. Mature osteoclasts were identified as described above. Images were taken by immunofluorescence microscope (Nikon, Tokyo, Japan).

\section{Quantitative Reverse-Transcription PCR (RT-qPCR)}

Osteoclasts were collected from BMMs that cultured with M-CSF (25 ng/mL) and RANKL (100 ng/mL) under various concentrations of sarsasapogenin $(0,1,2$, or $4 \mu \mathrm{M})$ for the dose-dependent effect of sarsasapogenin. RNAiso Plus RNA Extraction Reagent (Takara Bio, Japan) was used to isolate the total RNA from cells as previously described. cDNA (Complementary DNA) was synthesized using $1 \mu \mathrm{g}$ extracted RNA and Prime Script RT Master Mix (Takara Bio). The cDNA was used as a template for qPCR using SYBR Green qPCR Master Mix (Takara Bio) on an ABI Prism 7500 System (Applied Biosystems, CA, USA) under the following conditions: denaturation at $95^{\circ}$ $\mathrm{C}$ for $10 \mathrm{~min} ; 95^{\circ} \mathrm{C}$ for $10 \mathrm{~s}, 60^{\circ} \mathrm{C}$ for $20 \mathrm{~s}, 72^{\circ} \mathrm{C}$ for $20 \mathrm{~s}$, 40 cycles in total; $72^{\circ} \mathrm{C}$ for $90 \mathrm{~s}$ as the final extension. Specific primers of mouse are shown in Table 1.

\section{Western Blot Analysis}

To explore how sarsasapogenin affects the signaling pathways during osteoclast formation, cells were seeded in 6-well plates $\left(5 \times 10^{5}\right.$ cells/well $)$. To determine early RANKLinduced signal transduction events, cells were pretreated with $4 \mu \mathrm{M}$ sarsasapogenin for $1 \mathrm{~h}$, and then stimulated with RANKL (100 ng/mL) for $5,10,20,30$, or 60 minutes. To determine the late RANKL-induced signal transduction events, cells were treated with $100 \mathrm{ng} / \mathrm{mL}$ RANKL (without or without $4 \mu \mathrm{M}$ sarsasapogenin) for 1,3 or 5 days. The nontreated cells (time 0 ) served as negative control. RIPA lysis buffer (phosphatase and protease inhibitor cocktail contained) was used to extract the total cellular proteins. Lysates were centrifuged at $12,000 \mathrm{~g}$ for $15 \mathrm{~min}$ at $4^{\circ} \mathrm{C}$, and the supernatant was collected. Cytoplasmic and nuclear protein fractions were prepared using the NE-PER Nuclear and Cytoplasmic Extraction Kit (Thermo Fisher Scientific). Proteins were denatured in sodium dodecyl sulfatepolyacrylamide gel electrophoresis (SDS-PAGE) sampling buffer at $100^{\circ} \mathrm{C}$ for 5 minutes and $40 \mu \mathrm{g}$ of the total proteins were resolved by $10 \%$ SDS-PAGE. The separated proteins 
Table I Mouse Primers for qPCR

\begin{tabular}{|l|l|l|}
\hline Gene & Forward Primer & Reverse Primer \\
\hline$\beta$-Actin & $\begin{array}{l}\text { 5'-AGC CAT GTA CGT } \\
\text { AGC CAT CC-3' }\end{array}$ & $\begin{array}{l}\text { 5'-CTC TCA GCA GTG } \\
\text { GTG GTG AA-3' }\end{array}$ \\
\hline TRAP & $\begin{array}{l}\text { 5' -TCC TGG CTC AAA } \\
\text { AAG CAG TT-3' }\end{array}$ & $\begin{array}{l}\text { 5'-ACA TAG CCC ACA } \\
\text { CCG TTC TC-3' }\end{array}$ \\
\hline $\begin{array}{l}\text { CTSK } \\
\text { C-Fos }\end{array}$ & $\begin{array}{l}\text { 5'-CTT CCA ATA CGT } \\
\text { GCA GCA GA-3' }\end{array}$ & $\begin{array}{l}\text { 5'-TCT TCA GGG CTT } \\
\text { TCT CGT TC-3' }\end{array}$ \\
\hline NFATCI & $\begin{array}{l}\text { 5'-CAG GTC AAG AGC } \\
\text { 5'-AAG TAG TGC AGC } \\
\text { CAC TCT GGT C-3' }\end{array}$ & $\begin{array}{l}\text { 5'-CCC GGC TGC CTT } \\
\text { CCG TCT CAT A-3' }\end{array}$ \\
\hline $\begin{array}{l}\text { ATP6V0d2 } \\
\text { DC- } \\
\text { STAMP }\end{array}$ & $\begin{array}{l}\text { 5'-AAG CCT TTG TTT } \\
\text { GAC GCT GT-3' } \\
\text { 5'-CTT GCA ACC TAA }\end{array}$ & $\begin{array}{l}\text { 5'-TTC GAT GCC TCT } \\
\text { GTG AGA TG-3' } \\
\text { 5'-TCA ACA GCT CTG } \\
\text { TCG TGA CC-3' }\end{array}$ \\
\hline
\end{tabular}

were transferred to polyvinylidene fluoride (PVDF) membranes (Bio-Rad, California, USA). After blocking with 5\% $(\mathrm{w} / \mathrm{v})$ skimmed milk powder in TBST for 1 hour at room temperature $\left(20-25^{\circ} \mathrm{C}\right)$, the membranes and primary antibodies were incubated overnight at $4^{\circ} \mathrm{C}$. After washing three times with TBST, the membranes were incubated with horseradish peroxidase-conjugated secondary antibodies at room temperature for 2 hours. The bands were detected using BeyoECL Plus Enhanced Chemiluminescence Reagent (Beyotime Biotechnology Institute). ImageJ was used for optical density analysis.

\section{Immunofluorescence Detection of P65 Nuclear Translocation}

To test how sarsasapogenin affect the p65 nuclear translocation following RANKL stimulation, ${ }^{24}$ BMMs that seeded at $1 \times 10^{4}$ cells/well in a 12 -well plate were pretreated with $4 \mu \mathrm{M}$ sarsasapogenin for $30 \mathrm{~min}$. Then RANKL (100 ng/mL) was used to stimulate cells for $30 \mathrm{~min}$. The cells were fixed with 4\% PFA and washed gently with PBS. Non-specific immune response sites were blocked with 2\% BSA in PBS for 1 hour at room temperature. The cells were then incubated with anti-p65 antibody at $4^{\circ} \mathrm{C}$ overnight. After washing away the residual liquid with PBS, they were incubated with Alexa Fluor 647 labeled secondary antibody (Abcam, Cambridge, UK, UK) for 1 hour in the dark at room temperature. Nuclei were counterstained with DAPI for
5 min avoiding light. Images were acquired using an immunofluorescence microscope (Leica, Germany).

\section{Mouse Model of LPS-Induced Bone Loss}

To verify the therapeutic potential of sarsasapogenin, a mouse model of LPS-induced calvarial osteolysis was established. Animal care and use of laboratory animals were conducted in accordance with National Institutes of Health Guide for Care and Use of Laboratory Animals (NIH Pub No 85-23, revised 1996), and approved by the Zhejiang University Institutional Animal Care and Use Committee. Twenty-four 8-week-old C57/BL6 male mice were randomly divided into four groups: sham (PBS), LPS (5 mg LPS/kg and PBS), low-dose (5 mg LPS $/ \mathrm{kg}$ and $5 \mathrm{mg}$ sarsasapogenin $/ \mathrm{kg}$ ), and high-dose (5 mg LPS $/ \mathrm{kg}$ and $10 \mathrm{mg}$ sarsasapogenin $/ \mathrm{kg}$ ). ${ }^{17}$ The mice were injected subcutaneously on the sagittal midline suture of the calvarium under light anesthesia. Sarsasapogenin is a fatsoluble compound. Because of the long-term administration of osteoporosis patients, oral administration is appropriate. To ensure the accuracy of the dosage, the method of intragastric administration was used. According to the most commonly used method at present, sarsasapogenin was dissolved in sodium carboxymethyl cellulose solution to make a stable suspension for administration. One day before LPS (prophylactic treatment), PBS and sarsasapogenin were administered intragastrically and then injected every other day for 7 days. Finally, these mice were sacrificed. Following this, their calvaria were collected and put into 4\% PFA for further microcomputed tomography (micro-CT) and histological analysis.

\section{Micro-CT}

The high-resolution micro-CT was used to analyze the mouse calvariae under the conditions of isometric resolution of 18 $\mu \mathrm{m}$ and X-ray energy setting of $80 \mathrm{kV}$ and $100 \mu \mathrm{A}$. Threedimensional (3D) images were reconstructed by cone-beam reconstruction software (SkyScan, Altestel, Belgium). The analysis area of osteolysis-related indicators was the region of interest (ROI; $3 \times 3 \times 1 \mathrm{~mm}$ ) centered on the midline suture. CT analyzer software (SkyScan) was used to measure the ratio of bone volume to tissue volume (BV/TV, \%) and the number and area of holes in the ROI.

\section{Histological and Immunohistochemical Analysis}

Bone tissues from each group were fixed in 4\% PFA for 2 days and were then decalcified in $10 \%$ ethylenediaminetetraacetic acid for 7 days. They were subsequently embedded in paraffin 
and sectioned for morphometric examination by staining with hematoxylin and eosin (H\&E) and TRAP (Sigma-Aldrich) staining. $^{25}$ The sections were photographed using a microscope. Image-Pro Plus software (Media Cybernetics, Rockville, MD) was used to measure bone histomorphometric parameters such as BV/TV (\%), TRAP-positive osteoclasts number (number of osteoclasts on bone surface, N.OC/B.S), surface area of osteoclasts per bone surface (OCs/BS), etc.

\section{Statistical Analysis}

All data provided in this study were represented at least three independent experiments and the data were expressed as mean \pm SD. Statistical analysis was conducted by non-parametric test such as Mann Whitney test and Kruskal Wallis test in SPSS 19.0 (SPSS Inc., USA). Mann Whitney test was used to analyze the data of Western blot. Kruskal Wallis test was used to analyze the multi-sample data such as cytotoxicity, osteoclast formation and TRAP staining, F-actin and DAPI staining, bone resorption, RT-qPCR and all the data in vivo. When the multi-sample data had statistical significance, they were subjected to rank conversion for one-way analysis of variance (ANOVA). LSD was used as the ex-post comparison after one-way ANOVA. $P<0.05$ was judged to be statistically significant.

\section{Results}

\section{Sarsasapogenin Inhibited RANKL-Induced Osteoclast Formation in vitro}

To assess the cytotoxicity of sarsasapogenin on BMMs, the MTT assay was used. Cells were exposed to the indicated concentrations of sarsasapogenin $(1,2,4$, and 8 $\mu \mathrm{M}$ ) for 48 or $96 \mathrm{~h}$. No cytotoxic effect was observed, and BMMs remained viable at various time points for assessment of sarsasapogenin concentration below $4 \mu \mathrm{M}$ (Figure 1B). So $4 \mu \mathrm{M}$ was used as the upper limit concentration of sarsasapogenin for subsequent experiments. Next, doseand time-dependent effects of sarsasapogenin on osteoclast formation were tested. For dose-dependent analysis, cells were stimulated with RANKL, and various concentrations of sarsasapogenin for 6 days simultaneously. Large multinuclear "pancake" shape and obvious TRAP activity were used as the identification basis for mature osteoclasts. TRAP-positive osteoclast area and number decreased significantly with sarsasapogenin treatment in the dosedependent analysis (Figure 1C and D). To determine when sarsasapogenin exerts its inhibition, BMMs were stimulated with RANKL and processed with $4 \mu \mathrm{M}$ sarsasapogenin at each time point, as depicted in Figure 1E. Osteoclast formation was significantly inhibited at D0-D6, D0-D2, and D2-D4. TRAP-positive osteoclasts area and number decreased at D0-D6, D0-D2, and D2-D4 (Figure $1 F)$. Together, these results showed that sarsasapogenin inhibits osteoclast formation in a concentration-dependent manner and inhibition occurs early.

\section{Sarsasapogenin Inhibited F-Actin Ring and Bone Resorption in vitro}

Sarsasapogenin inhibited the large multinucleated osteoclasts formation, which shown in Figure 1C, suggesting the inhibitory effect on precursor cell fusion. To examine its inhibitory effects on F-actin rings formation and bone resorption, cells were treated with various sarsasapogenin doses. When treated with sarsasapogenin, the F-actin rings in osteoclasts showed a dose-dependent decrease. They also contained fewer nuclei per cell (Figure 2A and c). After treatment with $4 \mu \mathrm{M}$ sarsasapogenin, many cells appeared as monocytes (Figure 2A and C). Equal numbers of pre-osteoclasts (small osteoclasts) were sowed onto bovine discs. They were then stimulated with RANKL (100 ng/mL) and different doses of sarsasapogenin. Sarsasapogenin reduced the total resorption area dosedependently, especially in the presence of $4 \mu \mathrm{M}$ sarsasapogenin (Figure 2B and D). Together, these data indicated that sarsasapogenin inhibits bone resorption and F-actin ring formation.

\section{Sarsasapogenin Inhibited the Related Gene Expression of Osteoclast in vitro}

To elucidate the further inhibitory effect of sarsasapogenin on osteoclast formation, the mRNA expression levels of osteoclast-specific genes that are involved in osteoclast formation and bone resorption were measured by RTqPCR. With the stimulation of RANKL, several specific genes (including TRAP, CTSK, DC-STAMP, V-ATPased2, C-Fos, and NFATc1) were upregulated during osteoclast differentiation. ${ }^{26}$ Compared with the control group, due to the presence of sarsasapogenin, RANKL-induced osteoclast-related gene expression (including TRAP, CTSK, DC-STAMP, V-ATPase-d2, C-Fos and NFATc1) was strongly suppressed in a concentration-dependent manner (Figure 2E). These data showed that sarsasapogenin inhibits RANKL-induced osteoclast-related gene expression during differentiation in vitro. 

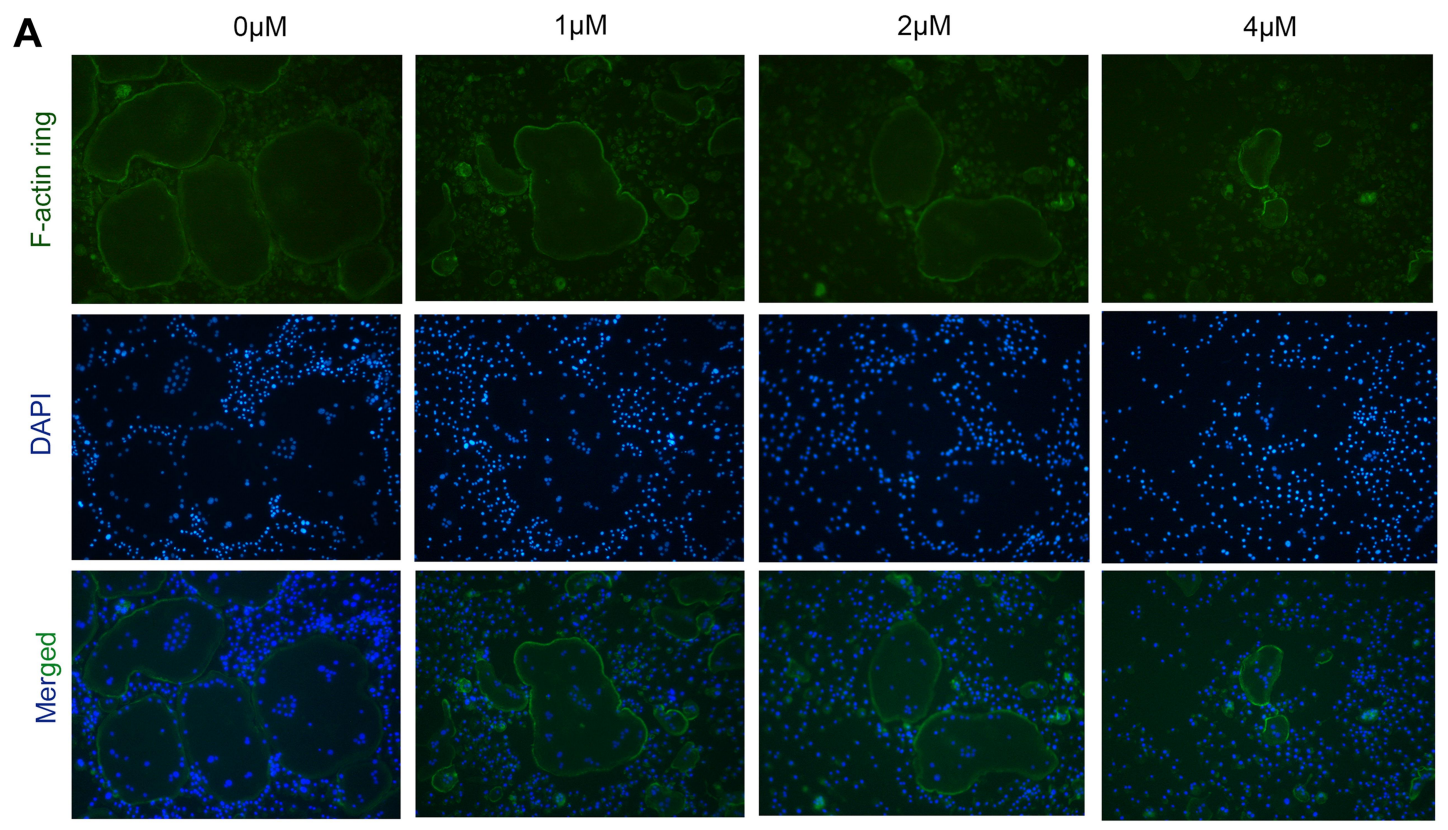

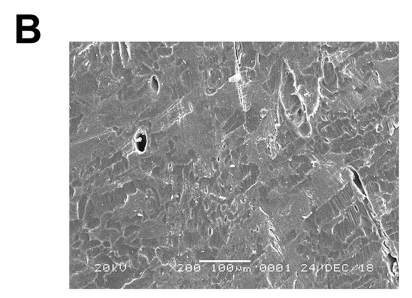

$0 \mu \mathrm{M}$

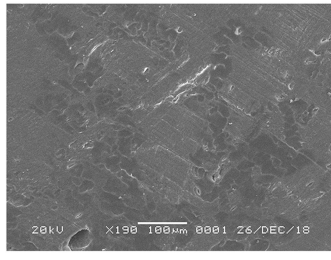

$1 \mu \mathrm{M}$

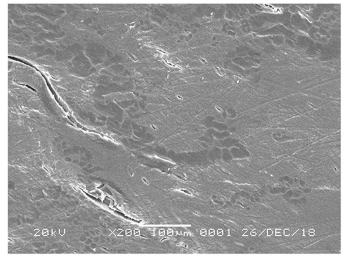

$2 \mu \mathrm{M}$

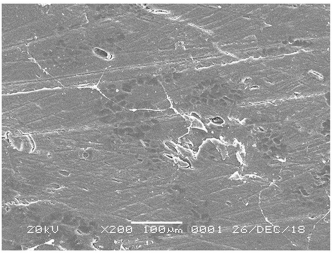

$4 \mu \mathrm{M}$
C

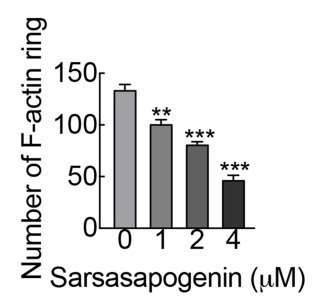

D

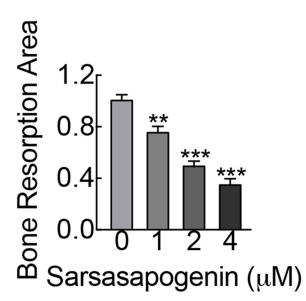

E
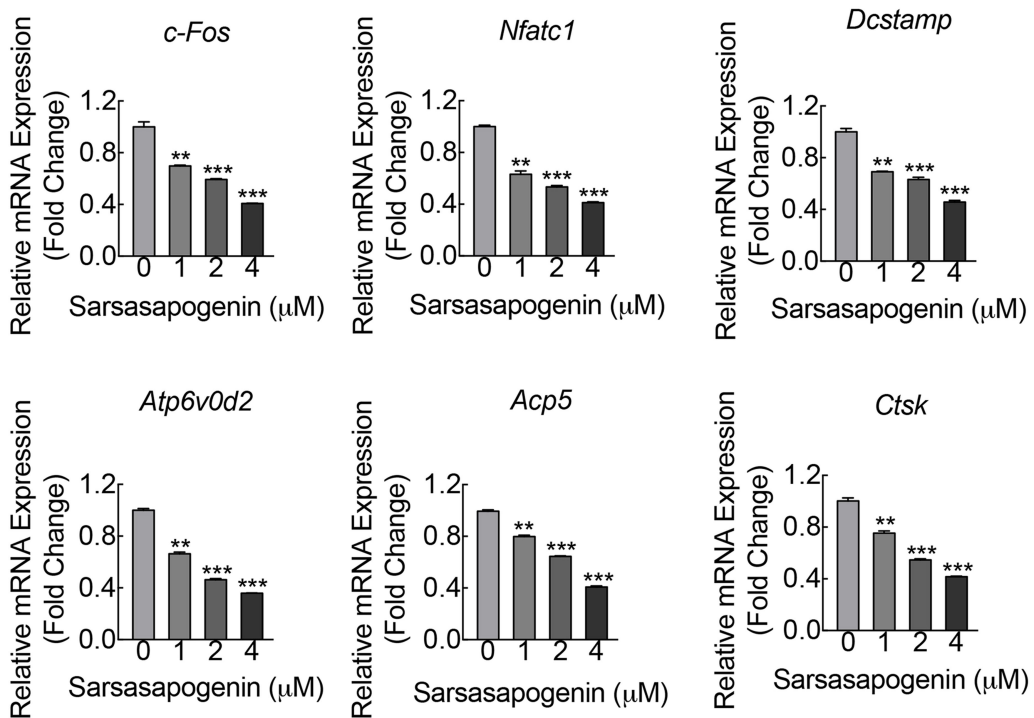

Figure 2 Sarsasapogenin inhibits F-actin ring and bone resorption in vitro. (A) Effect of sarsasapogenin on the fusogenic potential of osteoclast precursor cells. Representative immunofluorescence images of F-actin rings (Acti-stain ${ }^{\mathrm{TM}} 488$ Fluorescent Phalloidin; green) from BMM-derived osteoclasts stimulated with $100 \mathrm{ng} / \mathrm{mL}$ RANKL with or without the indicated concentrations of sarsasapogenin. Nuclei were counterstained with DAPI (blue) (magnification $=100 \times$; scale bar $=100 \mu \mathrm{m}$ ). $($ B) Equal numbers of pre-osteoclasts were seeded onto bone slices and stimulated with M-CSF and RANKL in the presence of different concentrations of sarsasapogenin. After 3 days, bone resorption lacunae were observed by SEM (magnification $=200 \times$; scale bar $=100 \mu \mathrm{m})$. (C) F-actin rings were quantified using Image $(n=3)$. (D) The resorption pit area $(\%)$ was quantified using Image $(n=3)$. $(E)$ Dose-dependent mRNA expression profile of osteoclast marker genes. RT-qPCR was conducted using RNA extracted from cells stimulated with $100 \mathrm{ng} / \mathrm{mL}$ RANKL with or without the indicated concentrations of sarsasapogenin for 5 days. mRNA levels of c-FOS, NFATcI, DC-STAMP, ATP6V0d2, TRAP, and CTSK were normalized to that of $\beta$-actin and then compared to RANKL-only control $(0)$ to obtain the relative fold change $(n=3)$. Bar graph presents the mean $\pm S D$. $* * p<0.01$, and $* * * p<0.00$ I versus RANKL-treated only control. 


\section{Sarsasapogenin Attenuated RANKL- Induced NF- $\kappa B$ and JNK/MAPK Signaling}

The early activation of the RANKL reactive signaling pathways are the important conditions for the induction of osteoclast formation. NF- $\mathrm{kB}, \mathrm{PI} 3 \mathrm{~K}-\mathrm{Akt}$, and MAPK pathways are the most important and earliest cascades activated in precursor BMMs after stimulation of RANKL. ${ }^{10,26,27}$ Rapid phosphorylation of Akt, ERK, p38 and JNK can be caused by RANKL stimulation, the latter three are the members of the MAPK cascade (Figure 3A, B and D). Phosphorylation of the aforementioned members is necessary for the activation of the downstream cascade during osteoclast formation. Relative to its total protein, pretreatment of BMM with sarsasapogenin could significantly inhibit JNK phosphorylation. However, sarsasapogenin treatment had no inhibitory effects on the phosphorylation of Akt, ERK, p38, and the total protein levels of PI3K (Figure 3A, B and D). Western blot analyses revealed that within the stimulation of RANKL for a few minutes, Iк $\beta \alpha$ degraded rapidly via the proteasomal degradation pathway (Figure $3 \mathrm{C}$ and D). Sarsasapogenin

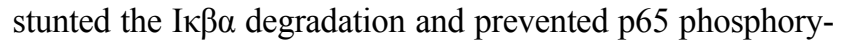
lation (Figure $3 \mathrm{C}$ and $\mathrm{D}$ ). Additionally, as seen with the immunofluorescence staining of p65, sarsasapogenin attenuated the transfer of p65 from the cytoplasm to the nucleus compared to the rate of transfer seen in the control group (Figure 3E and F). The induction of c-Fos is caused by the activation of NF- $\mathrm{KB}$ and MAPKs in response to RANKL. When c-Fos binds to c-JUN, they act as part of the AP-1 complex, leading to the activation of NFATc1, the major transcriptional activator of osteoclast formation. ${ }^{28}$ After RANKL stimulation, the protein expression of NFATc1 increased significantly, reached a peak on the third day, and then began to decrease. Sarsasapogenin treatment significantly weakened the NFATc1 induction protein expression in response to RANKL (Figure $3 \mathrm{G}$ and $\mathrm{H}$ ). Thus, these data indicated that sarsasapogenin suppresses RANKL-induced activation of the NF- $\mathrm{KB}$ and JNK/MAPK signaling pathways and thus attenuates NFATc1 induction.

\section{Sarsasapogenin Prevented LPS-Induced Bone Loss in vivo}

To investigate the potential protective effects of sarsasapogenin on pathological osteolysis, a murine model of LPS-induced bone loss was established. Relevant results showed that compared to the corresponding parameters in the sham group, BV/TV was significantly reduced and porosity was significantly increased in the LPS-treated group (Figure 4A-C). However, these decreases were significantly and dose-dependently inhibited by sarsasapogenin treatment (Figure 4A). Quantitative analysis of bone parameters showed that BV/TV increased significantly (Figure 4B), and porosity decreased significantly in concentration-dependent manner (Figure 4C). Histological and histomorphometric analysis further suggested that sarsasapogenin protects against LPS-induced osteolysis (Figure 5A-C). Further data showed that sarsasapogenin increased BV/TV (Figure 5B) and reduced the number of TRAP-positive cells in a dose-dependent manner (Figure 5C). To sum up, these data showed that sarsasapogenin prevents LPS-induced bone loss in vivo.

\section{Discussion}

Osteoclasts are giant polynuclear cells whose main function is bone resorption. Bone homeostasis is regulated by osteoclasts and osteoblasts. ${ }^{1}$ Enhanced bone resorption and osteoclast numbers in bone remodeling are manifestations of various osteolytic diseases, which pose serious health concern. Currently available anti-osteoporosis drugs often have severe side effects, including musculoskeletal pain, esophageal cancer, ocular inflammation, osteonecrosis of jaw, and atypical fractures. ${ }^{5-7}$ Therefore, there is an urgent need to develop safe alternative drugs that treat osteolytic diseases. In our study, we demonstrated that sarsasapogenin, a sapogenin present in the Chinese medical herb Anemarrhena asphodeloides Bunge, which exerts diverse biological actions, including anti-cancer and anti-inflammatory effects, ${ }^{16,17}$ can prevent bone loss and suppress osteoclastogenesis. Therefore, it has potential as a novel drug for osteoclast-related osteolytic diseases.

At the molecular level, the NF-KB and JNK/MAPK signaling pathways, which are downstream of RANKL and activated during osteoclastogenesis, were significantly inhibited by sarsasapogenin. Osteoclast differentiation is mainly modulated by RANKL-associated signaling pathways, including the PI3K/Akt, MAPK, and NF- $\mathrm{KB}$ pathways. The MAPK signaling pathway, including the transcription factor AP-1 (C-Fos and C-Jun), ${ }^{29}$ is indispensable to osteoclast formation because NFATc1 expression is regulated by AP-1. ${ }^{14,30-32}$ NFATC1 regulates osteoclast-related genes, including TRAP, CTSK, DCSTAMP, V-ATPase-d2, and C-Fos. ${ }^{33-36}$ Many plantderived drug monomer compositions have been found to inhibit osteoclastogenesis by suppressing MAPK signaling; for example, thymol, a natural monomer derived from thyme leaves, impairs osteoclast differentiation by this signaling. ${ }^{37}$ Myricitrin, a flavonoid glycoside derived 
A

A

p-ERK

ERK

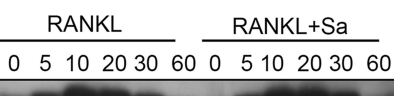

p-JNK

JNK

p-P38

P38

$\beta$-actin

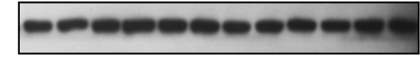

C

$$
\text { RANKL }
$$

$\mathrm{RANKL}+\mathrm{Sa}$

IкBa

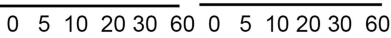

p-P65

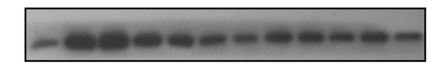

P65

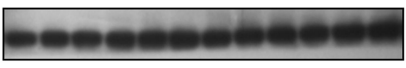

$\beta$-actin

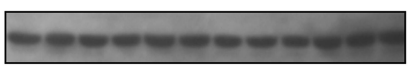

B

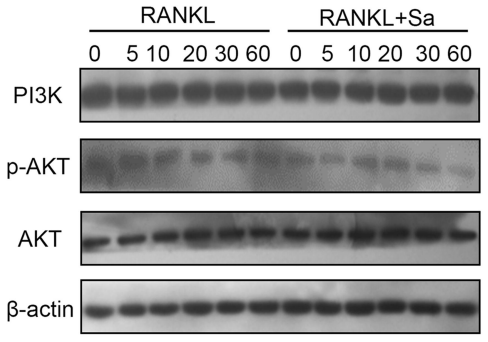

D
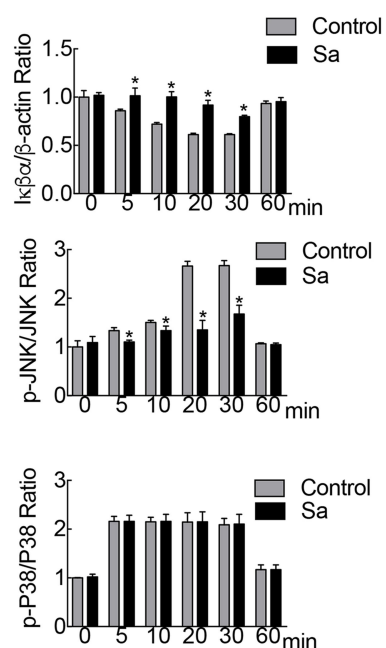
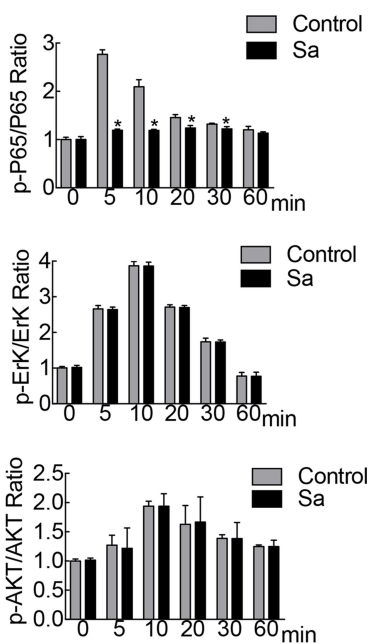

(E

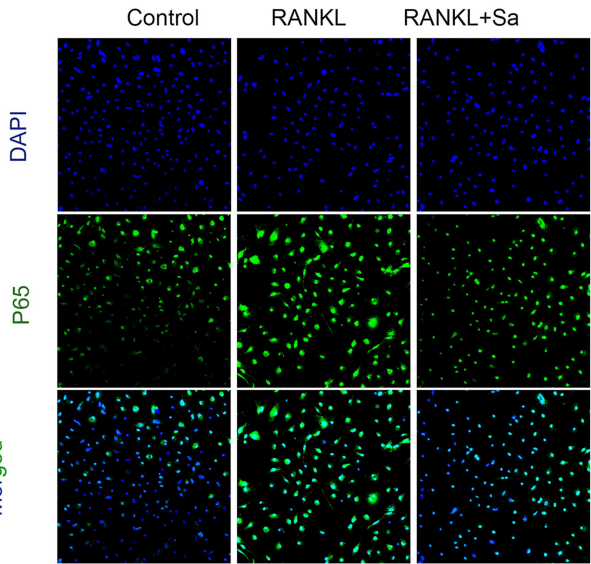

F

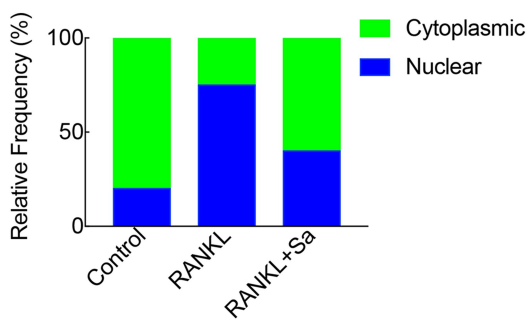

G
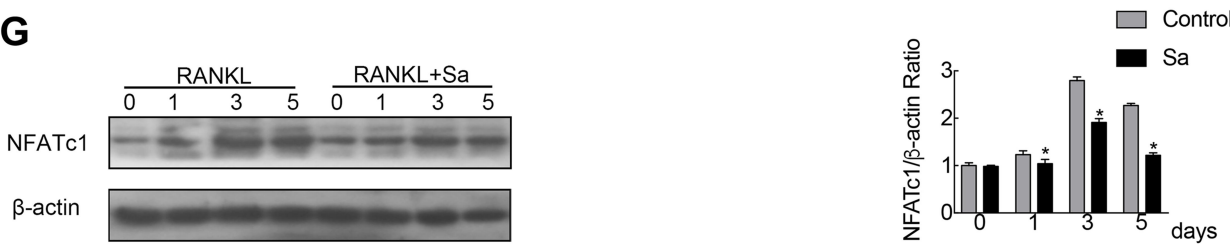

Figure 3 Sarsasapogenin attenuates RANKL-induced activation of the NF- $\kappa B$ and JNK/MPAK signaling pathways. (A-C) BMMs were pretreated with $4 \mu$ M sarsasapogenin for $\mathrm{I} h$ and then stimulated with RANKL for the indicated periods. Cell lysates were probed for protein levels using Western blot analysis $(\mathrm{n}=3$ ). (D) Relative changes in the phosphorylation statuses of $\mathrm{p} 65$, Akt, ERK, p38, and JNK were determined by densitometry and expressed as a ratio against its total protein counterpart, and the expression of I $\kappa \beta \alpha$ relative to actin, were determined using Imagej. (E) Sarsasapogenin prevents p65 nuclear translocation and localization. Representative immunofluorescence images of p65 localization (red) in BMMs treated with sarsasapogenin and stimulated with RANKL. Nuclei were counterstained with DAPI (blue). (F) The relative frequency of nuclear and cytoplasmic p65 under each experimental condition was quantified using Imagej $(n=3)$. (G) Total cellular proteins extracted from BMM-derived osteoclasts cotreated with RANKL and $4 \mu \mathrm{M}$ sarsasapogenin for $0, \mathrm{I}, 3$, or 5 days were subjected to immunoblot analyses using specific antibodies to NFATcl. $\beta$-Actin was used as an internal loading control. $(\mathrm{n}=3)$. Total BMM-derived osteoclasts were cultured with RANKL $(100 \mathrm{ng} / \mathrm{mL})$ and $\mathrm{M}-\mathrm{CSF}(25 \mathrm{ng} / \mathrm{mL})$ with or without sarsasapogenin $(4 \mu \mathrm{M})$ for 0 , I, 3 , or 5 days. Cell lysates were probed for NFATcl protein levels using Western blot analysis $(n=3)$. $(\mathbf{H})$ Relative expression of NFATcl was determined by densitometry and expressed as a ratio versus actin. Bar graphs represent the mean $\pm \mathrm{SD} ; \mathrm{*} p<0.05$ versus respective controls. 

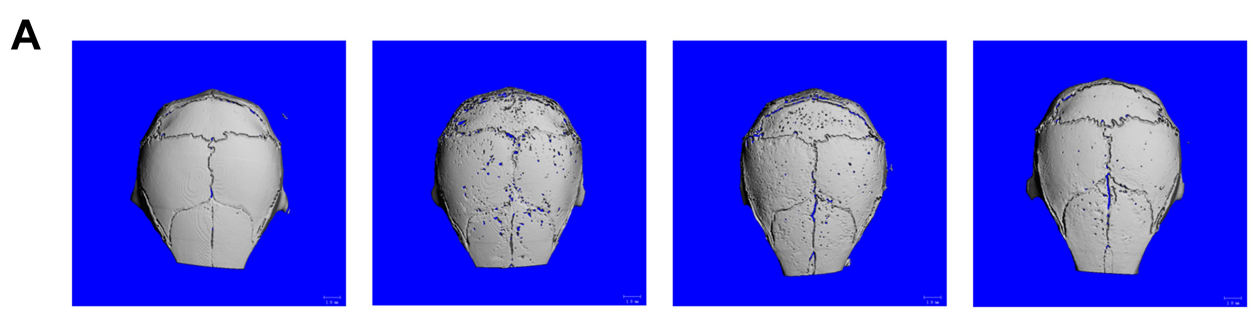

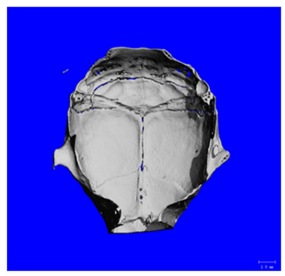

sham

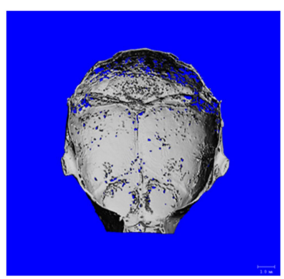

vehicle

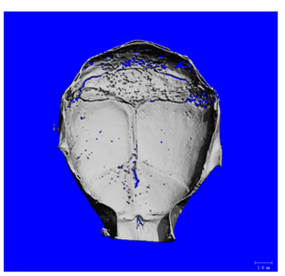

Low dose

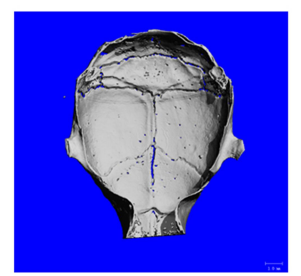

High dose
B

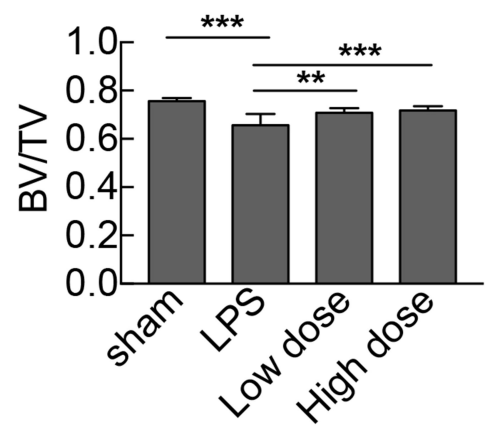

C

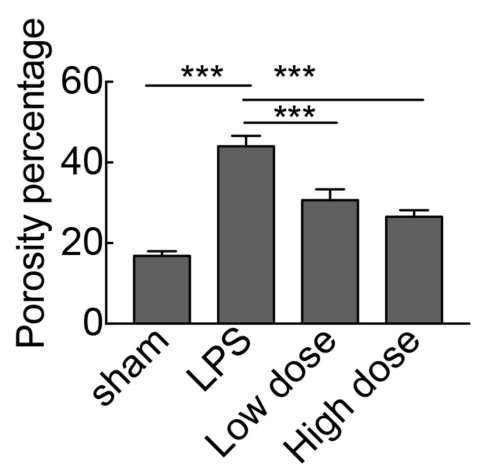

Figure 4 Sarsasapogenin protects against LPS-induced bone loss in vivo. (A) Micro-CT scanning and 3D reconstruction of the entire caldaria of mice from the sham (PBS), LPS ( $5 \mathrm{mg} \mathrm{LPS} / \mathrm{kg}$ body weight), low-dose sarsasapogenin ( $5 \mathrm{mg}$ LPS/ $\mathrm{kg}$ and $5 \mathrm{mg}$ sarsasapogenin/ $\mathrm{kg}$ ), and high-dose sarsasapogenin (5 mg LPS/kg and I0 mg sarsasapogenin/ $\mathrm{kg})$ groups $(\mathrm{n}=6)$. Scale bar $=$ I mm. (B) Quantitative analysis of BV/TV $(\mathrm{n}=6)$. (C) Percentage porosity $(\mathrm{n}=6)$. Data represent the mean \pm SD; $* * p<0.0 \mathrm{I}$ and $* * * p<0.00 \mathrm{I}$ versus respective controls.

from the roots of myrica, inhibits osteoclastogenesis through the MAPK signaling pathway. ${ }^{38}$ This is the first study to show that sarsasapogenin inhibits osteoclastogenesis by suppressing RANKL-induced JNK/MAPK signaling. In addition, we showed that sarsasapogenin inhibited NFATc1, downstream of the MAPK signaling pathway, in a time-based dependency. The combination of RANKL and RANK receptor triggers activates the NF- $\kappa B$ signaling, an important signaling pathway in osteoclast differentiation. ${ }^{10,14,39,40}$ Many plant-derived drug monomer compositions have been found to inhibit osteoclastogenesis, suppressing NF- $\mathrm{BB}$ : madecassoside, a monomer isolated from Centella asiatica, inhibits osteoclast differentiation, suppressing NF- $\mathrm{B}$ signaling and NFATc1 expression. $^{41}$ In the present study, we found that sarsasapogenin abrogated I $\beta$ degradation and suppressed p65 phosphorylation. Immunofluorescence staining and immunoblot analysis of cytoplasmic and nuclear fractions confirmed that sarsasapogenin inhibited the nuclear translocation of $\mathrm{p} 65$.

For the animal model section, LPS-induced bone loss is the most familiar model of osteoclast-related osteolytic diseases. $^{42}$ Therefore, we used the LPS-induced model to investigate whether sarsasapogenin would prevent bone loss. Sarsasapogenin is a fat-soluble compound. Because of the long-term administration of osteoporosis patients, oral administration is appropriate. To ensure the accuracy of the dosage, the method of intragastric administration was used. Sarsasapogenin prominently inhibited bone loss in this model. Morphometric analysis showed that sarsasapogenin significantly suppressed the reduction in BV/TV and an increased the porosity relative to the LPS group in a dosedependent manner, as shown by micro-CT scanning. Bone histomorphometry demonstrated that sarsasapogenin inhibited bone loss in vivo by reducing the number of TRAPpositive cells. 
A
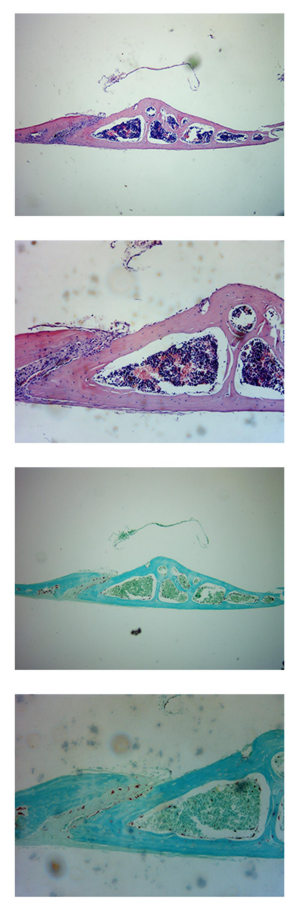

sham
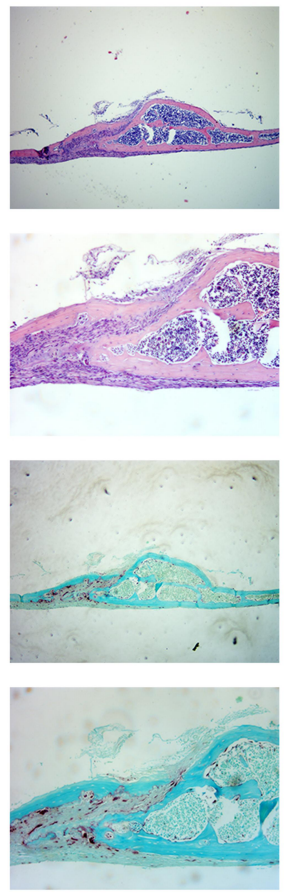

vehicle

B

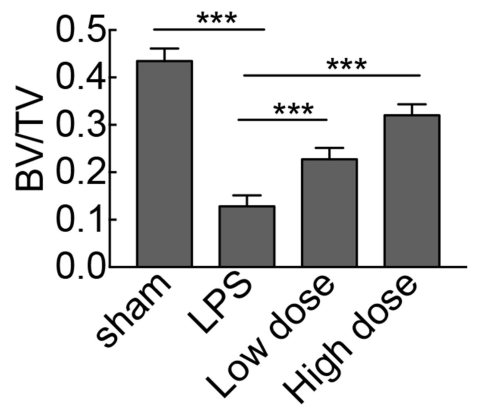

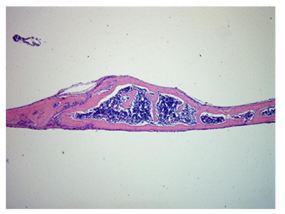
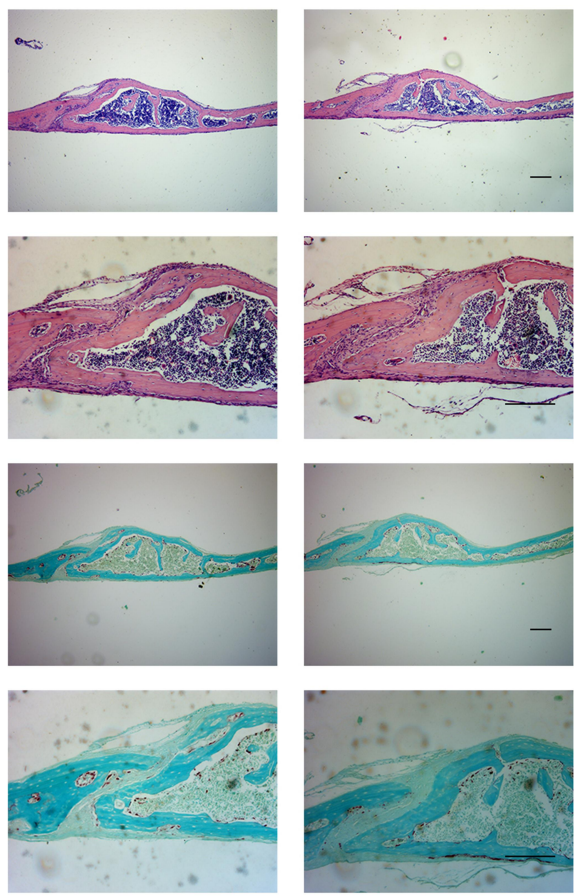

Low dose

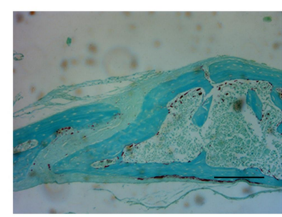

High dose

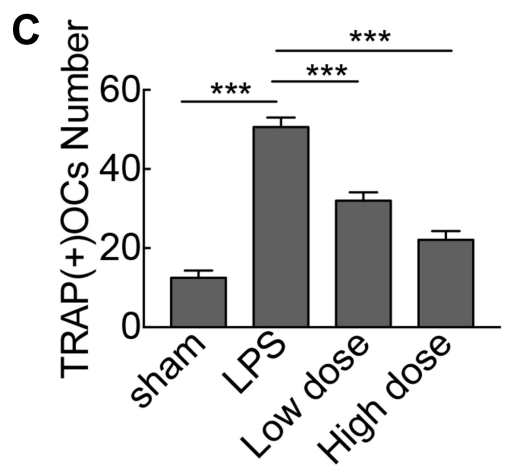

Figure 5 Histological and histomorphometric analyses of the effect of sarsasapogenin on LPS-induced bone loss in vivo. (A) Representative images of calvaria stained with H\&E and TRAP from the sham group (PBS; control), LPS (5 mg LPS/kg body weight), low-dose sarsasapogenin (5 mg LPS/kg and $5 \mathrm{mg}$ sarsasapogenin/kg), and high-dose sarsasapogenin group $(5 \mathrm{mg}$ LPS $/ \mathrm{kg}$ and $10 \mathrm{mg}$ sarsasapogenin $/ \mathrm{kg})(\mathrm{n}=6)($ scale bar $=200 \mu \mathrm{m}) .($ B and C) Quantification of BV/TV and TRAP $(+)$ cell number $(\mathrm{n}=6)$. Data represent the mean $\pm \mathrm{SD} ; * * * p<0.001$ versus respective controls.

Despite many new findings, there are also some limitations to our study and certain questions should be answered. First of all, although it is clear that sarsasapogenin exerts an inhibitory effect on the MAPK and NF- $\mathrm{kB}$ signaling pathways, the exact upstream molecular target needs to be further studied in order to understand its potential molecular mechanism better. Secondly, the complex relationships among osteoclasts, osteoblasts and osteocytes, the cell targets, and the biological characteristics of sarsasapogenin are not completely clear and need further exploration. Moreover, as for how sarsasapogenin reached the bone and regulated osteolysis, this involved the relevant knowledge of hemopharmacokinetics. We speculated that the mechanism of sarsasapogenin reaching the bone may be: after sarsasapogenin was absorbed by the digestive tract, it entered the blood circulation, thereby reaching bone tissue and affecting osteoclast differentiation. However, the exact mechanism still needs further pharmacokinetic research in the future.

In summary, our study shows that sarsasapogenin evidently protects against bone loss and inhibits osteoclastogenesis in vitro and in vivo through the NF- $\mathrm{kB}$ and JNK/ MAPK signaling pathways. Thus, together with the other biological functions of sarsasapogenin, we believe that sarsasapogenin has potential in the treatment of osteolytic diseases and can be used as a new therapeutic agent. 


\section{Statement}

According to previous research reports, after applying sarsasapogenin intervention, mice had no adverse reactions. ${ }^{17,21}$ Our observations also showed that after administration, the mice's various states such as drinking water and eating were normal, suggesting that this drug has no serious toxic reactions.

\section{Acknowledgments}

This study was supported by the National Science Foundation of China (Grant No. 81871801), Zhejiang Basic Public Welfare Research Project (LGF18H060010), Natural Science Foundation of Zhejiang Province (Grant No. LQ19H060001), Projects of Medical and Health Technology Development Program in Zhejiang Province (2018KY824), and Zhejiang Medical and Health Science and Technology Project (2019KY712).

\section{Disclosure}

Jiaxuan Peng, Kangxian Zhao and Jiling Zhu should be regarded as co-first authors.

The authors have no conflicts of interest.

\section{References}

1. Lorenzo J, Horowitz M, Choi Y. Osteoimmunology: interactions of the bone and immune system. Endocr Rev. 2008;29(4):403-440.

2. Hu B, Sun X, Yang Y, et al. Tomatidine suppresses osteoclastogenesis and mitigates estrogen deficiency-induced bone mass loss by modulating TRAF6-mediated signaling. FASEB J. 2019;33(2):2574-2586. doi:10.1096/fj.201800920R

3. Yuan G, Lian Z, Liu Q, et al. Phosphatidyl inositol 3-kinase (PI3K)-mTOR inhibitor PKI-402 inhibits breast cancer induced osteolysis. Cancer Lett. 2019;443:135-144. doi:10.1016/j.canlet.2018. 11.038

4. Jiang Y, Sang W, Wang C, et al. Oxymatrine exerts protective effects on osteoarthritis via modulating chondrocyte homoeostasis and suppressing osteoclastogenesis. J Cell Mol Med. 2018;22(8):3941-3954. doi: $10.1111 /$ jcmm. 13674

5. Black DM, Abrahamsen B, Bouxsein ML, Einhorn T, Napoli N. Atypical femur fractures: review of epidemiology, relationship to bisphosphonates, prevention, and clinical management. Endocr Rev. 2019;40(2):333-368. doi:10.1210/er.2018-00001

6. Wotton CJ, Green J, Brown A, et al. Use of oral bisphosphonates and risk of hospital admission with osteonecrosis of the jaw: large prospective cohort study in UK women. Bone. 2019;124:69-74. doi:10.1016/j.bone.2019.04.003

7. Kennel KA, Drake MT. Adverse effects of bisphosphonates: implications for osteoporosis management. Mayo Clin Proc. 2009;84(7):632637; quiz 638. doi:10.1016/S0025-6196(11)60752-0

8. Roodman GD. Cell biology of the osteoclast. Exp Hematol. 1999;27 (8):1229-1241. doi:10.1016/S0301-472X(99)00061-2

9. Arai F, Miyamoto T, Ohneda O, et al. Commitment and differentiation of osteoclast precursor cells by the sequential expression of C-Fms and receptor activator of nuclear factor $\mathrm{\kappa b}$ (rank) receptors. J Exp Med. 1999;190(12):1741. doi:10.1084/jem.190.12.1741
10. Takayanagi H. Osteoimmunology: shared mechanisms and crosstalk between the immune and bone systems. Nat Rev Immunol. 2007;7:292. doi:10.1038/nri2062

11. Mizukami J, Takaesu G, Akatsuka H, et al. Receptor activator of NF$\kappa \mathrm{B}$ ligand (RANKL) activates TAK1 mitogen-activated protein kinase kinase kinase through a signaling complex containing RANK, TAB2, and TRAF6. Mol Cell Biol. 2002;22(4):992-1000. doi:10.1128/MCB.22.4.992-1000.2002

12. Darnay BG, Besse A, Poblenz AT, Lamothe B, Jacoby JJ. TRAFs in RANK signaling. In: Wu H, editor. TNF Receptor Associated Factors (Trafs). New York: Springer New York; 2007:152-159.

13. Wong BR, Besser D, Kim N, et al. TRANCE, a TNF family member, activates Akt/PKB through a signaling complex involving TRAF6 and c-Src. Mol Cell. 1999;4(6):1041-1049. doi:10.1016/S1097-2765(00) 80232-4

14. Ha Kim J, Kim N. Regulation of NFATc1 in osteoclast differentiation. J Bone Metabol. 2014;21(4):233-241. doi:10.11005/ jbm.2014.21.4.233

15. Kim MS, Yang Y-M, Son A, et al. RANKL-mediated ROS pathway that induces long-lasting $\mathrm{Ca} 2+$ oscillations essential for osteoclastogenesis. J Biol Chem. 2010;285(10):6913-6921.

16. Shen S, Zhang Y, Zhang R, Gong X. Sarsasapogenin induces apoptosis via the reactive oxygen species-mediated mitochondrial pathway and ER stress pathway in HeLa cells. Biochem Biophys Res Commun. 2013;441(2):519-524. doi:10.1016/j.bbrc.2013.10. 101

17. Lim SM, Jeong JJ, Kang GD, Kim KA, Choi HS, Kim DH. Timosaponin AIII and its metabolite sarsasapogenin ameliorate colitis in mice by inhibiting NF- $\mathrm{KB}$ and MAPK activation and restoring Th17/Treg cell balance. Int Immunopharmacol. 2015;25(2):493-503. doi:10.1016/j.intimp.2015.02.016

18. Ma D, Zhang J, Sugahara K, Sagara Y, Kodama H. Effect of sarsasapogenin and its derivatives on the stimulus coupled responses of human neutrophils. Clin Chim Acta. 2001;314(1-2):107-112. doi:10.1016/S0009-8981(01)00638-6

19. Park BK, So KS, Ko HJ, et al. Therapeutic potential of the rhizomes of anemarrhena asphodeloides and timosaponin A-III in an animal model of lipopolysaccharide-induced lung inflammation. Biomol Ther (Seoul). 2018;26(6):553-559. doi:10.4062/biomolther.2017. 249

20. Bao W, Pan H, Lu M, Ni Y, Zhang R, Gong X. The apoptotic effect of sarsasapogenin from Anemarrhena asphodeloides on HepG2 human hepatoma cells. Cell Biol Int. 2007;31(9):887-892. doi:10. 1016/j.cellbi.2007.02.001

21. Dong D, Zhou NN, Liu RX, et al. Sarsasapogenin-AA13 inhibits LPS-induced inflammatory responses in macrophage cells in vitro and relieves dimethylbenzene-induced ear edema in mice. Acta Pharmacol Sin. 2017;38(5):699-709. doi:10.1038/aps.2016. 180

22. Huang C, Dong D, Jiao Q, Pan H, Ma L, Wang R. SarsasapogeninAA13 ameliorates $A \beta$-induced cognitive deficits via improving neuroglial capacity on $\mathrm{A} \beta$ clearance and antiinflammation. CNS Neurosci Ther. 2017;23(6):498-509. doi:10.1111/cns.12697

23. Zhang T, Zhao K, Han W, et al. Deguelin inhibits RANKL-induced osteoclastogenesis in vitro and prevents inflammation-mediated bone loss in vivo. J Cell Physiol. 2019;234(3):2719-2729. doi:10.1002/ jcp. 27087

24. Nich C, Langlois J, Marchadier A, et al. Oestrogen deficiency modulates particle-induced osteolysis. Arthritis Res Ther. 2011;13(3): R100. doi:10.1186/ar3381

25. Yang $\mathrm{H}, \mathrm{Xu} \mathrm{Y}$, Zhu M, et al. Inhibition of titanium-particle-induced inflammatory osteolysis after local administration of dopamine and suppression of osteoclastogenesis via D2-like receptor signaling pathway. Biomaterials. 2016;80:1-10. doi:10.1016/j.biomaterials.2015.11.046

26. Boyle WJ, Simonet WS, Lacey DL. Osteoclast differentiation and activation. Nature. 2003;423:337. doi:10.1038/nature01658 
27. Takayanagi H. Mechanistic insight into osteoclast differentiation in osteoimmunology. J Mol Med. 2005;83(3):170-179. doi:10.1007/ s00109-004-0612-6

28. Takayanagi H, Kim S, Koga T, et al. Induction and activation of the transcription factor NFATc1 (NFAT2) integrate RANKL signaling in terminal differentiation of osteoclasts. Dev Cell. 2002;3(6):889-901. doi:10.1016/S1534-5807(02)00369-6

29. Wagner EF. Bone development and inflammatory disease is regulated by AP-1 (Fos/Jun). Ann Rheum Dis. 2010;69(Suppl 1):i86. doi:10.1136/ard.2009.119396

30. Grigoriadis A, Wang Z, Cecchini M, et al. c-Fos: a key regulator of osteoclast-macrophage lineage determination and bone remodeling. Science. 1994;266(5184):443-448. doi:10.1126/science.7939685

31. Kashiwada M, Shirakata Y, Inoue J-I, et al. Tumor necrosis factor receptor-associated factor 6 (TRAF6) stimulates extracellular signal-regulated kinase (ERK) activity in CD40 signaling along a Ras-independent pathway. J Exp Med. 1998;187(2):237-244. doi:10.1084/jem.187.2.237

32. Khosla S. Minireview: the OPG/RANKL/RANK system. Endocrinology. 2001;142(12):5050-5055. doi:10.1210/endo.142.12.8536

33. Cackowski FC, Anderson JL, Patrene KD, et al. Osteoclasts are important for bone angiogenesis. Blood. 2010;115(1):140. doi:10.1182/blood-2009-08-237628

34. Kim K, Lee S-H, Ha Kim J, Choi Y, Kim N. NFATc1 induces osteoclast fusion via up-regulation of Atp6v0d2 and the dendritic cell-specific transmembrane protein (DC-STAMP). Mol Endocrinol. 2008;22(1):176-185. doi:10.1210/me.2007-0237

35. Angel NZ, Walsh N, Forwood MR, Ostrowski MC, Cassady AI, Hume DA. Transgenic mice overexpressing tartrate-resistant acid phosphatase exhibit an increased rate of bone turnover. $J$ Bone Miner Res. 2000;15(1):103-110. doi:10.1359/jbmr.2000.15.1.103
36. Gelb BD, Shi G-P, Chapman HA, Desnick RJ. Pycnodysostosis, a lysosomal disease caused by cathepsin K deficiency. Science. 1996;273(5279):1236. doi:10.1126/science.273.5279.1236

37. Sapkota M, Li L, Kim S-W, Soh Y. Thymol inhibits RANKL-induced osteoclastogenesis in RAW264.7 and BMM cells and LPS-induced bone loss in mice. Food Chem Toxicol. 2018;120:418-429. doi:10.1016/j.fct.2018.07.032

38. Wang B, Hao D, Zhang Z, et al. Inhibition effects of a natural inhibitor on RANKL downstream cellular signalling cascades cross-talking. J Cell Mol Med. 2018;22(9):4236-4242. doi:10.1111/ jcmm.13703

39. Kim J-M, Lee J-H, Lee G-S, et al. Sophorae Flos extract inhibits RANKL-induced osteoclast differentiation by suppressing the NF$\kappa \mathrm{B} / \mathrm{NFATc} 1$ pathway in mouse bone marrow cells. BMC Complement Altern Med. 2017;17(1):164. doi:10.1186/s12906-016-1550-x

40. Zhai ZJ, Li HW, Liu GW, et al. Andrographolide suppresses RANKL-induced osteoclastogenesis in vitro and prevents inflammatory bone loss in vivo. Br J Pharmacol. 2014;171(3):663-675. doi:10.1111/bph. 12463

41. Wang Q, Yao L, Xu K, et al. Madecassoside inhibits estrogen deficiency-induced osteoporosis by suppressing RANKL-induced osteoclastogenesis. J Cell Mol Med. 2019;23(1):380-394.

42. Wu Z, Wu H, Li C, et al. Daphnetin attenuates LPS-induced osteolysis and RANKL mediated osteoclastogenesis through suppression of ERK and NFATc1 pathways. J Cell Physiol. 2019;234 (10):17812-17823. doi:10.1002/jcp.28408
Drug Design, Development and Therapy

\section{Publish your work in this journal}

Drug Design, Development and Therapy is an international, peerreviewed open-access journal that spans the spectrum of drug design and development through to clinical applications. Clinical outcomes, patient safety, and programs for the development and effective, safe, and sustained use of medicines are a feature of the journal, which has also been accepted for indexing on PubMed Central. The manuscript management system is completely online and includes a very quick and fair peer-review system, which is all easy to use. Visit http://www. dovepress.com/testimonials.php to read real quotes from published authors. 